\title{
CORRIGENDUM
}

\section{Sources, isolation, characterisation and evaluation of probiotics - CORRIGENDUM}

\author{
Luis Fontana, Miriam Bermudez-Brito, Julio Plaza-Diaz, Sergio Muñoz-Quezada and Angel Gil \\ (First published online 19 September 2013)
}

doi:10.1017/S0007114512004011, Published by Cambridge University Press, 29 January 2013.

The British Journal of Nutrition and Cambridge University Press have investigated an alert to possible plagiarism in the article by Fontana L, Bermudez-Brito M, Plaza-Diaz J, Muñoz-Quezada S and Gil A published in the British Journal of Nutrition (2013; 109, Suppl. 2: S35-50). The investigation found that there was a minor duplication of text from an article by Girardin and Seidman published in Digestive Diseases (2011; 29: 574-87). Specifically, sentences in the abstracts of both the articles were found to be identical. There was no further duplication in the rest of the text or in any other part of the article. Consequently, the alert to possible plagiarism was upheld. In accordance with the Committee on Publication Ethics guidelines (http://www. publicationethics.org), Fontana et al. ${ }^{(1)}$ were requested to rewrite the abstract of their article. The revised abstract is given below.

\begin{abstract}
According to the FAO and the WHO, probiotics are 'live microorganisms which, when administered in adequate amounts, confer a health benefit on the host'. The strains most frequently used as probiotics include lactic acid bacteria and bifidobacteria, which are isolated from traditional fermented products and the gut, faeces and breast milk of human subjects. The identification of microorganisms is the first step in the selection of potential probiotics. The present techniques, including genetic fingerprinting, gene sequencing, oligonucleotide probes and specific primer selection, discriminate closely related bacteria with varying degrees of success. Additional molecular methods, such as denaturing gradient gel electrophoresis/temperature gradient gel electrophoresis and fluorescence in situ hybridisation, are employed to identify and characterise probiotics. The ability to examine fully sequenced genomes has accelerated the application of genetic approaches to the elucidation of the functional roles of probiotics. One of the best-demonstrated clinical benefits of probiotics is the prevention and treatment of acute and antibiotic-associated diarrhoea; however, there is mounting evidence for a potential role for probiotics in the treatment of allergies and intestinal, liver and metabolic diseases. There are various mechanisms by which probiotics exert their beneficial effects: regulation of intestinal permeability, normalisation of host intestinal microbiota, improvement of gut immune barrier function, and adjustment between pro- and anti-inflammatory cytokines. The number of studies carried out to test the effects of probiotics in vitro and in animals is enormous. However, the most reliable method of assessing the therapeutic benefits of any probiotic strain is the use of randomised, placebo-controlled trials, which are reviewed in this article.
\end{abstract}

\section{Reference}

1. Fontana L, Bermudez-Brito M, Plaza-Diaz J, et al. (2013) Sources, isolation, characterisation and evaluation of probiotics. BrJ Nutr 109, S35-S50, Published by Cambridge University Press, 29 January 2013, doi:10.1017/S0007114512004011. 